\title{
A Study of Intermediate Predicate Logics
}

By

\author{
Hiroakira $\mathrm{ONO}^{*}$
}

As stated in the survey [3] by Hosoi and the author, many works have been done during the last decade on the propositional logics between the classical and the intuitionistic (the intermediate propositional logics). On the other hand, only a little progress has been made in the study of intermediate predicate logics except Umezawa's pioneering work [12] and some recent works [13], [14]. In the present paper, we shall develop a study of intermediate predicate logics.

We want to study intermediate logics in a general framework, intending to derive some properties common to many intermediate logics, as we mentioned already in [8]. Semantical methods have played an important role in doing so. But, for the intermediate predicate logics they are found to be incomplete. Indeed, we have shown in [9] and [10] that we can use neither Kripke's semantics nor the algebraic one as a uniform way of studying intermediate predicate logics. These incompleteness results present a limitation of semantical methods, but nevertheless there are many problems we can solve now. In this paper we shall prove some basic results on the intermediate predicate logics and their models. We emphasize here that some of them can be easily extended to modal predicate logics and logics stronger than the minimal predicate logics.

We define intermediate predicate logics in $\S 1$. In $\S 2$ and $\S 3$, two kinds of semantics for them are introduced and some basic properties of them are proved. In $\S 4$, we study the cardinality of models and show analogues of Löwenheim theorem. We prove some syntactical results in

Received September 28, 1972.

* This work is partly supported by the Sakkokai Foundation. 
$\S 5$. In $\S 6$, we show a certain relationship between predicate logics and propositional logics. We believe that this is a typical example of a general study of logics.

Finally, we would like to stress that without Umezawa's work we could not have any clear insight.

\section{§1. Intermediate Predicate Logics}

First of all, we fix a language $\mathscr{L}$ in order to define intermediate predicate logics. $\mathscr{L}$ consists of a list of countably infinite individual variables $x, y, z$ etc. and a list of countably infinite $n$-ary predicate variables $p^{(n)}, q^{(n)}, r^{(n)}$ etc. for each non-negative integer $n$. 0 -ary predicate variables are identified with propositional variables. Occasionally, we omit the superscript letter on a predicate variable. $\mathscr{L}$ contains no constants and no function symbols. The logical symbols of $\mathscr{L}$ are $\wedge, \vee, \rightarrow, \neg, \forall$ and $\exists$. Formulas (of first order) are defined in the usual way. The letters $A, B, C$ etc. will denote them. A formula containing neither quantifiers nor predicate variables except propositional variables is called a propositional formula. As we mention below, we identify a logic with the set of formulas provable in it. Thus, by $L K$ (or $L J$ ), we mean the set of formulas provable in the pure classical (or intuitionistic) predicate calculus. (See Church $[1]$.)

Definition 1.1. A set of formulas $L$ is called an intermediate predicate logic if it satisfies the following four conditions:

1) $L J \subset L \subset L K$.

2) $L$ is closed under modus ponens.

3) $L$ is closed under the generalization. That is, if a formula $A$ is in $L$ then for any individual variable $x(\forall x) A$ is also in $L$.

4) $L$ is closed under the substitution. That is, if a formula $A$ is in $L$ and $x_{1}, \cdots, x_{n}$ are distinct individual variables then any formula of the form $\check{S}_{B}^{\left(x_{1}, \cdots, x_{n}\right)} A \mid$ is also in L. (As for the definition of $\check{S}$, see [1].)

We notice that if $L$ is an intermediate predicate logic then it is 
closed under the alphabetic change of bound or free variables, since it contains $L J$.

For each intermediate predicate logic $L$, the set of propositional formulas in $L$ is an intermediate propositional logic in the sense of Hosoi [2]. We call it the propositional fragment of $L$ and write it as $\pi(L)$. Let

$$
L K_{\pi}=\pi(L K) \text { and } L J_{\pi}=\pi(L J) .
$$

Then $L K_{\pi}$ (or $L J_{\pi}$ ) is the classical (or the intuitionistic) propositional logic.

Throughout this paper we sometimes call an intermediate predicate (or propositional) logic only as a predicate (or a propositional) logic. $\mathscr{I}$ (or $\mathscr{I}_{\pi}$ ) is the set of all the predicate (or the propositional) logics. Hosoi [2] introduced the lattice operations $\cap$ and $\cup$ on $\mathscr{I}_{\pi^{*}}$ In the same way, we can extend them on $\mathscr{I}$. Then the following theorem holds.

Theorem 1.2. $\mathscr{I}$ is a complete pseudo-Boolean lattice with respect to $\cap$ and $\cup$.

By Jankov's result [4], we have immediately that $\mathscr{I}$ contains $2{ }^{0}$ logics.

Let $H$ be any set of formulas and $L$ be any predicate logic. Then we write $L+H$ for the smallest set of formulas which contains any formula either in $L$ or in $H$ and is closed under modus ponens, the generalization and the substitution. Therefore, if $H C L K$ then $L+H$ is a predicate logic. Similarly, if $H$ is a set of propositional formulas and $K$ is a propositional logic, $K+H$ denotes the smallest set of propositional formulas which contains any formula either in $K$ or in $H$ and is closed under modus ponens and the substitution (of propositional formulas). Sometimes, we write $L+A_{1}+\cdots+A_{n}$ for $L+\left\{A_{1}, \cdots, A_{n}\right\}$. A predicate logic $L$ is called to be finitely axiomatizable if there is a finite subset $H$ of $L K$ such that $L=L J+H$. 


\section{§2. The Algebraic Semantics}

In this and the next $\S \S$, we shall define two kinds of semantics and study their basic properties. As we deal only with logics and not with theories, we would say structures (or frames) as models, opposing the usual usage of the word model. For instance, as a model of the classical logic, we would take the 2 -valued Boolean algebra with a countable domain, not considering a fixed interpretation of each predicate variable. So, as for the algebraic semantics, we get the following definition.

A pseudo-Boolean algebra $P$ is said to be $\lambda$-complete for some cardinal $\lambda$, if both $\bigcap_{a \in S} a$ and $\bigcup_{a \in S} a$ exist in $P$ for any subset $S$ of $P$ such that $\overline{\bar{S}} \leqq \lambda$.

Definition 2.1 $(P, V)$ is called a pseudo-Boolean model (a p.B. model) if $V$ is a nonempty set (a universe) and $P$ is a $\lambda$-complete (nondegenerate) pseudo-Boolean algebra, where $\lambda$ is the cardinality of $V$.

Let $(P, V)$ be a p.B. model. An assignment $f$ of $(P, V)$ is a function such that

1) $f(p) \in P$ for each propositional variable $p$,

2) $f\left(p^{(n)}\right)$ is a function from $V^{n}$ to $P$ for each $n$-ary predicate variable $p^{(n)}(n>0)$.

Now, we add a constant $\vec{v}$ for each element $v$ in $V$ to the original language $\mathscr{L} . \mathscr{L}[V]$ is the extended language thus obtained. For each assignment $f$, define a function $f^{\prime}$ from the set of closed formulas in $\mathscr{L}[V]$ to $P$ by the following rules;

$$
\begin{aligned}
& f^{\prime}(p)=f(p), \\
& f^{\prime}\left(p^{(n)}\left(\bar{v}_{1}, \cdots, \bar{v}_{n}\right)\right)=f\left(p^{(n)}\right)\left(v_{1}, \cdots, v_{n}\right), \\
& f^{\prime}(A \wedge B)=f^{\prime}(A) \cap f^{\prime}(B), \\
& f^{\prime}(A \vee B)=f^{\prime}(A) \cup f^{\prime}(B),
\end{aligned}
$$




$$
\begin{aligned}
& f^{\prime}(A \rightarrow B)=f^{\prime}(A) \supset f^{\prime}(B), \\
& f^{\prime}(\neg A)=-f^{\prime}(A), \\
& f^{\prime}(\forall x A(x))=\bigcap_{v \in V} f^{\prime}(A(\bar{v})), \\
& f^{\prime}(\exists x A(x))=\bigcup_{v \in V} f^{\prime}(A(\bar{v})),
\end{aligned}
$$

where $\cap, \cup, \supset$ and - denote the corresponding lattice operations of $P$. Hereafter, we identify this $f^{\prime}$ with $f$. We write $1_{P}$ (or $0_{P}$ ) for the greatest (or the least) element of $P$.

A formula $A$ of $\mathscr{L}$ is said to be valid in a p.B. model $(P, V)$ if $f\left(A^{\prime}\right)=1_{P}$ for each assignment $f$ of $(P, V)$, where $A^{\prime}$ is a closure of $A$. $L^{+}(P, V)$ denotes the set of formulas valid in $(P, V)$. It is easy to see that the set $L^{+}(P, V)$ contains $L J$ and is closed under each of three operations mentioned in Definition 1.1. The following theorem shows that there is a predicate logic which cannot be characterized by a single p.B. model.

Theorem 2.2 ([10]). There exists a predicate logic $L$ such that 1$)$ $L=L^{+}\left(P_{1}, V_{1}\right) \cap L^{+}\left(P_{2}, V_{2}\right)$ for some p.B. models $\left(P_{i}, V_{i}\right)(i=1,2)$ but 2) $L \neq L^{+}(P, V)$ for any p.B. model $(P, V)$.

This fact suggests the following definition.

Definition 2.3 ([10]). A set of p.B. models $\left\{\left(P_{i}, V_{i}\right) ; i \in I\right\}$ is said to be characteristic for a predicate logic $L$, if $L=\bigcap_{i \in I} L^{+}\left(P_{i}, V_{i}\right)$. In this case, we say that $L$ has a characteristic set of p.B. models $\left\{\left(P_{i}, V_{i}\right)\right.$; $i \in I\}$.

Theorem 2.4 ([10]). There exist $2 \$ 0$ predicate logics having no characteristic sets of p.B. models.

Now, we shall give a condition for a set $\bigcap_{i \in I} L^{+}\left(P_{i}, V_{i}\right)$ to be an intermediate predicate logic. Formulas $F_{1}, F_{2}$ and $F i n$ are defined as follows: 


$$
\begin{aligned}
F_{1}= & \forall x r(x, x) \wedge \forall x \forall y(r(x, y) \vee r(y, x)) \\
& \wedge \forall x \forall y \forall z(r(x, y) \wedge r(y, z) \rightarrow r(x, z)), \\
F_{2}= & \neg \forall x \exists y \neg r(y, x), \\
F i n= & F_{1} \rightarrow F_{2} .
\end{aligned}
$$

The intuitive meaning of Fin will be obvious. We can expect that a model which validates $F i n$ has a finite universe. Of course, Fin $\notin L K$.

Theorem 2.6. $\bigcap_{i \in I} L^{+}\left(P_{i}, V_{i}\right)$ is an intermediate predicate logic if and only if there is $a j \in I$ such that $V_{j}$ is infinite.

Proof. First, suppose that $V_{j}$ is infinite. To show that $\bigcap_{i \in I} L^{+}\left(P_{i}, V_{i}\right)$ is an intermediate predicate logic, it suffices to show that $\bigcap_{i \in I}^{i \in I} L^{+}\left(P_{i}, V_{i}\right)$ CLK. Clearly,

$$
\bigcap_{i \in I} L^{+}\left(P_{i}, V_{i}\right) \subset L^{+}\left(P_{j}, V_{j}\right)
$$

The model $\left(P_{j}, V_{j}\right)$ contains the submodel $\left(S_{1}, V_{j}\right)$, where $S_{1}$ denotes the 2 -valued Boolean algebra. Thus,

$$
L^{+}\left(P_{j}, V_{j}\right) \subset L^{+}\left(S_{1}, V_{j}\right)=L K
$$

So, $\bigcap_{i \in I} L^{+}\left(P_{i}, V_{i}\right) \subset L K$. Next, we shall show that the formula Fin is valid in a p.B. model $(P, V)$ if $V$ is finite. Suppose otherwise. Then there is an assignment $f$ of $(P, V)$ such that $f(F i n) \neq 1_{P}$. Let $f\left(F_{1}\right)=a$ and $f\left(F_{2}\right)=b$. Since $a$ and $\|b\|$ is the greatest element of $P / F-\left\{1_{P \mid F}\right\}$, where $P / F$ denotes the quotient algebra of $P$ with respect to $F$ and \|\| denotes the natural mapping from $P$ to $P / F$. Define an assignment $g$ of the p.B. model $(P / F, V)$ by

$$
g\left(r\left(\bar{v}_{1}, \bar{v}_{2}\right)\right)=\left\|f\left(r\left(\bar{v}_{1}, \bar{v}_{2}\right)\right)\right\| \quad \text { for any } v_{1}, v_{2} \in V .
$$

Since $V$ is finite, we can prove that $g\left(F_{1}\right)=\|a\|=1_{P / F}$ and $g\left(F_{2}\right)=\|b\|$. Define a relation $\leqq$ on $V$ by 


$$
v \leqq v^{\prime} \text { if and only if } g\left(r\left(\bar{v}, \bar{v}^{\prime}\right)\right)=1_{P \mid F} .
$$

Then, we can prove that the relation $\leqq$ is a quasi-order on $V$ and that either $v \leqq v^{\prime}$ or $v^{\prime} \leqq v$ holds for any $v, v^{\prime} \in V$, as for any $\|c\|,\|d\| \in P / F$ if $\|c\| \cup\|d\|=1_{P \mid F}$ then either $\|c\|=1_{P \mid F}$ or $\|d\|=1_{P \mid F} . \quad$ As $V$ is finite, there is a $v_{0}$ in $V$ such that $v \leqq v_{0}$ holds for any $v \in V$. That is,

$$
g\left(r\left(\bar{v}, \bar{v}_{0}\right)\right)=1_{P \mid F} \quad \text { for any } v \in V .
$$

Hence, $g\left(F_{2}\right)=g(\neg \forall x \exists y \neg r(y, x))=1_{P \mid F} \neq\|b\|$. This is a contradiction. Thus, if $V$ is finite then $F i n \in L^{+}(P, V)$. Now, suppose that $V$ is finite for each $i \in I$. Then

$$
\text { Fin } \in \bigcap_{i \in I} L^{+}\left(P_{i}, V_{i}\right)
$$

Hence $\bigcap_{i \in I} L^{+}\left(P_{i}, V_{i}\right) \not \subset L K$.

When we study propositional logics, the decomposition theorem plays an important role (see [3]). We have used its analogue for p.B. models with a finite universe in the above proof. Consider the following proposition (2.7), which means the decomposition theorem for p.B. models.

(2.7) For any p.B. model $(P, V)$, there is a set of p.B. models $\left\{\left(P_{i}, V_{i}\right)\right.$; $i \in I\}$ such that

1) $L^{+}(P, V)=\bigcap_{i \in I} L^{+}\left(P_{i}, V_{i}\right)$ and

2) each $P_{i}$ is irreducible, i.e., $P_{i}-\left\{1_{P_{i}}\right\}$ has the greatest element.

Theorem 2.8. (2.7) does not hold.

Proof. Let

$$
P=\{(x, y) ; 0 \leqq x<\omega, y=0,1\} \cup\{(\omega, 1)\}
$$

and $\quad V=\{n ; n<\omega\}$.

$P$ is ordered by a relation $\leqq *$ such that

$$
\left(x_{1}, y_{1}\right) \leqq *\left(x_{2}, y_{2}\right) \text { if } x_{1} \geqq x_{2} \text { and } y_{1} \geqq y_{2} \text {. }
$$

Then $P$ forms a complete pseudo-Boolean algebra with respect to $\leqq *$. 
Thus $(P, V)$ is a p.B. model. This model is nothing but Model 9 in Umezawa [12]. We can show that the formula $(p \rightarrow q) \vee(q \rightarrow p)$ is valid in $(P, V)$. It is shown in [12] that the following formula Dis (which means a distributive law)

$$
\text { Dis; } \quad \forall x(p(x) \vee q) \rightarrow(\forall x p(x) \vee q)
$$

is not valid in $(P, V)$. Now suppose that (2.7) holds. Then there exist p.B. models $\left(P_{i}, V_{i}\right)(i \in I)$ such that $L^{+}(P, V)=\bigcap_{i \in I} L^{+}\left(P_{i}, V_{i}\right)$ and each $P_{i}$ is irreducible. Since

$$
(p \rightarrow q) \vee(q \rightarrow p) \in L^{+}\left(P_{i}, V_{i}\right) \quad \text { for } i \in I,
$$

$P_{i}$ must be a linear pseudo-Boolean algebra for $i \in I$. But then $D i s$ is valid in $L^{+}\left(P_{i}, V_{i}\right)$ for each $i \in I$. This is a contradiction.

On the other hand, we show later that the decomposition theorem for Kripke models holds.

\section{§3. Kripke's Semantics}

We shall discuss Kripke's semantics in the present §. Many of basic properties of Kripke models for propositional logics can be naturally extended.

Definition 3.1. ( $M, U)$ is called a Kripke model if $M$ is a nonempty partially ordered set (with the order $\leqq_{M}$ ) and $U$ is a function from $M$ to the power set of $a$ set $S$ such that 1$)$ if $a \leqq_{M} b$ then $U(a) \subset U(b)$ and 2) $U(a)$ is nonempty for any $a \in M$.

We sometimes omit the subscript $M$ of $\leqq_{M}$. A function $W$ is said to be a valuation of a Kripke model $(M, U)$ if it satisfies the following conditions.

Let $a$ and $b$ be arbitrary elements in $M$. Then

1) for any propositional variable $p, W(p, a) \in\{t, f\}$ and if $a \leqq b$ and $W(p, a)=t$ then $W(p, b)=t$, 
2) for any $n$-ary $(n>0)$ predicate variable $p^{(n)}$,

$$
W\left(p^{(n)}, a\right) \subset U(a)^{n} \quad \text { and }
$$

if $a \leqq b$ then $W\left(p^{(n)}, a\right) \subset W\left(p^{(n)}, b\right)$.

Now, corresponding to each valuation $W$, we define a function $W^{\prime}$ which takes a value $t$ or $f$ for each pair $(A, a)$ of a closed formula $A$ in the language $\mathscr{L}\left[\bigcup_{b \in M} U(b)\right]$ and an element $a$ of $M$ by the following rules. $\left(\mathscr{L}\left[\bigcup_{b \in M} U(b)\right]\right.$ denotes the language which is obtained from $\mathscr{L}$ by adding a constant $\bar{u}$ for any element $u$ in $\bigcup_{b \in M} U(b)$.)

$$
\begin{aligned}
& W^{\prime}(p, a)=t \text { iff } W(p, a)=t \\
& W^{\prime}\left(p^{(n)}\left(\bar{u}_{1}, \cdots, \bar{u}_{n}\right), a\right)=t \text { iff }\left(u_{1}, \cdots, u_{n}\right) \in W\left(p^{(n)}, a\right), \\
& W^{\prime}(A \wedge B, a)=t \text { iff } W^{\prime}(A, a)=t \text { and } W^{\prime}(B, a)=t \\
& W^{\prime}(A \vee B, a)=t \text { iff } W^{\prime}(A, a)=t \text { or } W^{\prime}(B, a)=t \\
& W^{\prime}(A \rightarrow B, a)=t \text { iff } W^{\prime}(A, b)=f \text { or } W^{\prime}(B, b)=t
\end{aligned}
$$

for any $b$ such that $a \leqq b$,

$$
W^{\prime}(\neg A, a)=t \text { iff } W^{\prime}(A, b)=f \quad \text { for any } b \text { such that } a \leqq b \text {, }
$$

$W^{\prime}(\forall x A(x), a)=t$ iff $W^{\prime}(A(\bar{u}), b)=t \quad$ for any $b$ such that $a \leqq b$ and for any $u \in U(b)$,

$$
W^{\prime}(\exists x A(x), a)=t \text { iff } W^{\prime}(A(\bar{u}), a)=t \quad \text { for some } u \in U(a) .
$$

Hereafter, we identify $W^{\prime}$ with $W$. A formula $A$ of $\mathscr{L}$ is said to be valid in $(M, U)$ if for any valuation $W$ of $(M, U)$ and for any $a \in M$, $W\left(A^{\prime}, a\right)=t$, where $A^{\prime}$ is a closure of $A$. We write $L(M, U)$ for the set of formulas valid in a $\operatorname{Kripke}$ model $(M, U)$. If $L=L(M, U)$ for some Kripke model $(M, U)$ we say that $L$ has a characteristic Kripke model $(M, U)$. The following three theorems can be proved quite similarly as Corollary 2.8, Theorem 2.10 and Theorem 2.11 of [7]. They are fundamental in the succeeding discussions. 
Theorem 3.2. For any set of Kripke models $\left\{\left(M_{i}, U_{i}\right) ; i \in I\right\}$, there is a Kripke model $(M, U)$ such that $L(M, U)=\bigcap_{i \in I} L\left(M_{i}, U_{i}\right)$.

Compare the above theorem with Theorem 2.2. Now, suppose that $(M, U)$ is a Kripke model. For each $a \in M$, let $M_{a}=\left\{b ; a \leqq_{M} b\right\}$ and $U_{a}$ be the restriction of $U$ to $M_{a}$. Then $\left(M_{a}, U_{a}\right)$ is a Kripke model. It is easy to see that

$$
L(M, U)=\bigcap_{a \in M} L\left(M_{a}, U_{a}\right)
$$

Thus we have the following decomposition theorem for Kripke models (cf. Theorem 2.8).

Theorem 3.3. For any Kripke model $(M, U)$ there exists a set of Kripke models $\left\{\left(M_{i}, U_{i}\right) ; i \in I\right\}$ such that

1) $L(M, U)=\bigcap_{i \in I} L\left(M_{i}, U_{i}\right)$,

2) each $M_{i}$ has the least element.

Suppose that $(M, U)$ and $\left(N, U^{\prime}\right)$ are arbitrary Kripke models and that there are functions $f$ and $g$ such that

1) $f$ is an embedding of $M$ into $N$ (see [7]),

2) $g$ is a function from $\bigcup_{a \in M} U(a)$ to $\bigcup_{b \in M} U^{\prime}(b)$ such that $g(U(a))$ $=U^{\prime}(f(a))$ for each $a \in M$. Then we say that $(M, U)$ is embeddable into $\left(N, U^{\prime}\right)$.

Theorem 3.4. If $(M, U)$ is embeddable into $\left(N, U^{\prime}\right)$, then $L(M, U)$ $C L\left(N, U^{\prime}\right)$

Corollary 3.5. Suppose that $(M, U)$ and $\left(N, U^{\prime}\right)$ are Kripke models satisfying the following conditions;

1) there is an embedding of $M$ into $N$,

2) there is a set $S$ such that $U^{\prime}(b)=S$ for any $b \in N$ and $\overline{\overline{U(a)}} \geqq \overline{\bar{S}}$ for any $a \in M$.

Then $L(M, U) \subset L\left(N, U^{\prime}\right)$. 
In the following, we shall give a condition for a set $L(M, U)$ to be an intermediate predicate logic (cf. Theorem 2.6).

Theorem 3.6. $L(M, U)$ is an intermediate predicate logic if and only if there is an element $a \in M$ such that $U(a)$ is infinite.

Proof. At first, we remark that for any Kripke model $(M, U), L(M, U)$ contains $L J$ and is closed under each of three operations in Definition 1.1. Thus it suffices to show that $L(M, U) \subset L K$, in order to prove that $L(M, U)$ is an intermediate predicate logic. Suppose first that $U(a)$ is finite for any $a \in M$. We show that the formula Fin is valid in $L(M, U)$. Suppose not. Then there exists a valuation $W$ such that

$$
W(\text { Fin, } a)=f \quad \text { for some } a \in M \text {. }
$$

Thus, there is a $b$ such that

$$
\text { 1) } a \leqq b, \quad \text { 2) } W\left(F_{1}, b\right)=t \text { and } W\left(F_{2}, b\right)=f \text {. }
$$

By 2), there is a $c$ such that $b \leqq c$ and

$$
W(\forall x \exists y \neg r(y, x), c)=t .
$$

Thus, for each $u \in U(c)$ there is a $u^{\prime} \in U(c)$ such that

$$
W\left(r\left(\bar{u}^{\prime}, \bar{u}\right), c\right)=f
$$

Define a binary relation $\varliminf^{*}$ on $U(c)$ by

$$
u \leqq * u^{\prime} \text { if and only if }\left(u, u^{\prime}\right) \in W(r, c)
$$

By 2$), \leqq^{*}$ is a quasi-order on $U(c)$ and either $u \leqq^{*} u^{\prime}$ or $u^{\prime} \leqq^{*} u$ holds for each $u, u^{\prime} \in U(c)$. Since $U(c)$ is finite, there is a $u^{*} \in U(c)$ such that $u \leqq * u^{*}$ holds for any $u \in U(c)$. By the definition, this implies that $W\left(r\left(\bar{u}, \bar{u}^{*}\right), c\right)=t$ holds for any $u \in U(c)$. This is a contradiction. Thus we have that $\operatorname{Fin} \in L(M, U)$.

Next suppose that $U(a)$ is infinite for some $a$ in $M$. Then $L(M, U)$ $C L\left(M_{a}, U_{a}\right)$ where the model $\left(M_{a}, U_{a}\right)$ is defined in the same way as in 
the paragraph just above Theorem 3.3. Suppose that $N$ is a singleton set $\{b\}$ and $U^{\prime}$ is a function such that $U^{\prime}(b)=\{n ; n<\omega\}$. Let $f$ be a (constant) function from $M_{a}$ to $N$. Then by Corollary 3.5,

$$
L\left(M_{a}, U_{a}\right) \subset L\left(N, U^{\prime}\right)=L K
$$

Thus $L(M, U)$ is an intermediate predicate logic.

Remark. Let $F i n^{\prime}$ be the formula $F_{1} \rightarrow \exists x \forall y r(y, x)$, where $F_{1}$ is the formula introduced in (2.5). Then, we can show similarly as Theorem 2.6 that $F i n^{\prime} \in L^{+}(P, V)$ if and only if $V$ is finite. On the other hand, it does not hold always that $F i n^{\prime} \in L(M, U)$ if and only if $U(a)$ is finite for any $a \in M$. This follows from the fact that the predicate logic (but not intermediate) $L J+F i n$ has no characteristic sets of p.B. models. In other words, $F i n^{\prime} \notin L J+F i n$, but there are no p.B. models $(P, V)$ such that

$$
\text { Fin } \in L^{+}(P, V) \text { and } F i n^{\prime} \notin L^{+}(P, V) \text {. }
$$

It follows from this, for instance, that any predicate logic of the form $L J+\left\{A_{i} \vee F i n ; i \in I\right\}$, where each $A_{i}$ is a propositional formula not in $L J_{\pi}$, has no characteristic sets of p.B. models.

Theorem 3.7 ([9]). There exist $2 \$ 0$ predicate logics having no characteristic Kripke models.

We have remarked in $[10]$ that there is a predicate logic having a characteristic set of p.B. models but not having a characteristic Kripke model and that there is a predicate logic having a characteristic Kripke model but not having a characteristic set of p.B. models.

Now, we show how the formula Dis plays an important role in the proof of Theorem 1 in [10]. The following theorem can be proved similarly as Corollary 1.31 ) of [7].

Theorem 3.8. If Dis is in a predicate logic $L$ which has a characteristic Kripke model, then $L$ has a characteristic set of p.B. models. 


\section{§4. Cardinality of Models}

We shall discuss the cardinality of models in this $\S$. In the definition of Kripke models, the range of the function $U$ is the power set of some set $S$. Is it necessary to consider the case where $S$ is a very large set? A well-known result related to this problem is Löwenheim theorem for the classical predicate logic [6]. We will prove a kind of Löwenheim theorem for predicate logics characterized by either type of models.

Theorem 4.1. For any Kripke model $(M, U)$ such that $\overline{\bar{M}}<\overline{\overline{\bigcup_{a \in M} U(a)}}$, there is a Kripke model $\left(M, U^{\prime}\right)$ such that

1) $L\left(M, U^{\prime}\right)=L(M, U)$,

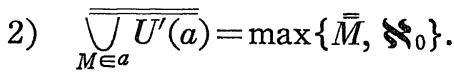

Proof. We assume that $\bigcup_{a \in M} U(a)$ is well-ordered. For each $a \in M$, we write $u_{a}$ for the first element of $U(a)$ with respect to the well-ordering above mentioned. We take an enumeration $\left\{A_{n} ; n<\omega\right\}$ of all the closed formulas not valid in $(M, U)$. For each $A_{n}$, we take a valuation $W_{n}$ of $(M, U)$ such that $W_{n}\left(A_{n}, a\right)=f$ for some $a \in M$. Now, for each $k<\omega$ we define a set $H_{n}^{(k)}(n<\omega)$ of closed formulas in the language $\mathscr{L}\left[\bigcup_{a \in M} U(a)\right]$ and a subset $V^{(k)}$ of $\bigcup_{a \in M} U(a)$ by induction on $k$.

$k=0: \quad H_{n}^{(0)}=\left\{A_{n}\right\} \quad$ for each $n<\omega$.

$$
V^{(0)}=\left\{u_{a} ; a \in M\right\} .
$$

$k=m+1$ : Assume that we have defined $H_{n}^{(m)}$ and $V^{(m)}$. For each $B \in$ $H_{n}^{(m)}$, we define first a subset $S_{(B, n)}$ of $M$ by

$$
S_{(B, n)}= \begin{cases}\left\{c \in M ; W_{n}(B, c)=t\right\} & \text { if } B \text { is of the form } \exists x C(x), \\ \left\{c \in M ; W_{n}(C(\bar{u}), c)=f\right. & \text { for some } u \in U(c)\} \\ \text { empty } & \text { if } B \text { is of the form } \forall x C(x), \\ \text { otherwise. }\end{cases}
$$


For each $c \in S_{(B, n)}$, define an element $u(B, n, c)$ of $U(c)$ by

$$
u(B, n, c)=\left\{\begin{array}{r}
\text { the first element of }\left\{u ; u \in U(c) \text { and } W_{n}(c(\bar{u}), c)=t\right\} \\
\text { with respect to the well-ordering if } B \text { is of the } \\
\text { form } \exists x C(x), \\
\text { the first element of }\left\{u ; u \in U(c) \text { and } W_{n}(C(\bar{u}), c)=f\right\} \\
\text { with respect to the well-ordering if } B \text { is of the } \\
\text { form } \forall x C(x) .
\end{array}\right.
$$

Now, define $V^{(m+1)}$ by

$$
V^{(m+1)}=V^{(m)} \cup\left\{u(B, n, c) ; n<\omega, B \in H_{n}^{(m)} \text { and } c \in S_{(B, n)}\right\} .
$$

Next, we define a set of formulas $H_{n}^{(m+1)}$ by the smallest set $H$ satisfying the following conditions. For each $B \in H_{n}^{(m)}$,

1) $B \in H$,

$2)$ if $B$ is of any one of the forms, $C \wedge D, C \vee D, C \rightarrow D$ then both $C$ and $D$ are in $H$,

3) if $B$ is of the form ${ }_{\neg} C$ then $C$ is in $H$,

4) if $B$ is of the form $\forall x C(x)$ then

4.1) if $W_{n}(B, c)=t$ then $C(\bar{u})$ is in $H$ for any $u \in U(c) \cap V^{(m+1)}$,

4.2) if $c \in S_{(B, n)}$ then $C(\overline{u(B, n, c)})$ is in $H$,

$5)$ if $B$ is of the form $\exists x C(x)$ then

5.1) if $c \in S_{(B, n)}$ then $C(\overline{u(B, n, c)})$ is in $H$,

5.2) otherwise $C(\bar{u})$ is in $H$ for any $u \in U(c) \cap V^{(m+1)}$.

Let

$$
V=\bigcup_{k<\omega} V^{(k)} \text { and } H_{n}=\bigcup_{k<\omega} H_{n}^{(k)}
$$

It is easy to see that each formula in $H_{n}$ is in the language $\mathscr{L}\left[\bigcup_{a \in M} U(a) \cap V\right]$.

Lemma 4.2. $\overline{\bar{V}}=\max \left\{\overline{\bar{M}}, \aleph_{0}\right\}$.

Now, we define a Kripke model $\left(M, U^{\prime}\right)$ by

$$
U^{\prime}(a)=U(a) \cap V \quad \text { for any } a \in M \text {. }
$$


Since $V$ is a subset of $\bigcup_{a \in M} U(a), \bigcup_{a \in M} U^{\prime}(a)=V$. Thus $\overline{\overline{\bigcup_{a \in M} U^{\prime}(a)}}=\max \{\overline{\bar{M}}$, $\left.\$ \$_{0}\right\}$ by the above lemma.

Leamma 4.3. $L(M, U) \subset L\left(M, U^{\prime}\right)$.

Proof. From the proof of Theorem 3.3, it follows that

$$
L(M, U)=\bigcap_{a \in M} L\left(M_{a}, U_{a}\right)
$$

Let $U^{\prime}{ }_{a}$ be the restriction of $U^{\prime}$ to $M_{a}$. Then we have also that

$$
L\left(M, U^{\prime}\right)=\bigcap_{a \in M} L\left(M_{a}, U_{a}^{\prime}\right)
$$

Thus, it suffices to prove that

$$
L\left(M_{a}, U_{a}\right) \subset L\left(M_{a}, U_{a}^{\prime}\right) \quad \text { for each } a \in M
$$

Define a function $g$ from $\bigcup_{b \in M_{a}} U_{a}(b)$ to $\bigcup_{b \in M_{a}} U_{a}^{\prime}(b)$ by

$$
g(u)= \begin{cases}u & \text { if } u \in V \\ u_{a} & \text { otherwise }\end{cases}
$$

Then $g\left(U_{a}(b)\right)=U_{a}^{\prime}(b)$ for any $b \in M$. By Theorem 3.4,

$$
L\left(M_{a}, U_{a}\right) \subset L\left(M_{a}, U_{a}^{\prime}\right)
$$

Lemma 4.4. $L\left(M, U^{\prime}\right) \subset L(M, U)$.

Proof. Suppose that $A$ is a closed formula not valid in $(M, U)$. We shall show that $A$ is not valid in $\left(M, U^{\prime}\right)$. Since $A \notin L(M, U), A=A_{n}$ for some $n<\omega$. We define a valuation $W_{n}^{\prime}$ by

1) $W_{n}^{\prime}(p, a)=W_{n}(p, a)$ for any $a \in M$ and any propositional variable $p$,

2) $W_{n}^{\prime}\left(q^{(m)}, a\right)=W_{n}\left(q^{(m)}, a\right) \cap U^{\prime}(a)^{m} \quad$ for any $a \in M$ and any $m$-ary predicate variable $q^{(m)}(m>0)$.

Now, we show that if a formula $B$ is in $H_{n}$, then 


$$
W_{n}^{\prime}(B, a)=W_{n}(B, a) \quad \text { for any } a \in M,
$$

by induction on the number of logical symbols appearing in $B$. When $B$ is atomic or of any one of the forms $C \wedge D, C \vee D, C \rightarrow D$ and $\neg C$, (4.5) can be easily ascertained by using the definition and the hypothesis of induction. Suppose that $B$ is $\exists x C(x)$. Let $W_{n}(B, a)=t$. Then

$$
u(B, n, a) \in U(a) \text { and } W_{n}(C(\overline{u(B, n, a)}), a)=t
$$

by the definition. If $B$ is in $H_{n}^{(k)}$ then $u(B, n, a) \in V^{(k+1)}$. Thus $C(\overline{u(B, n, a)}) \in H_{n}^{(k+1)}$. By the hypothesis of induction, $W_{n}^{\prime}(C(\overline{u(B, n, a)})$, $a)=t$. So, $W_{n}^{\prime}(B, a)=t$. Conversely, let $W_{n}(B, a)=f$. Then

$$
W_{n}(C(\bar{u}), a)=f \quad \text { for any } u \in U^{\prime}(a) .
$$

If $u \in U^{\prime}(a)$ then $u \in V^{(h)}$ for some $h<\omega$. Put $k=\max \{h-1, j\}$, where $j$ is the smallest integer $i$ such that $B \in H_{n}^{(i)}$. Then $u \in V^{(k+1)}$ and $B \in$ $H_{n}^{(k)}$. So, $C(\bar{u}) \in H_{n}^{(k+1)} \subset H_{n}$. Thus by the hypothesis,

$$
W_{n}^{\prime}(C(\bar{u}), a)=f \quad \text { for any } u \in U^{\prime}(a) .
$$

Hence,

$$
W_{n}^{\prime}(B, a)=f \text {. }
$$

When $B$ is of the form $\forall x C(x),(4.5)$ can be proved similarly as the above case. Now our theorem follows from lemmata $4.2,4.3$ and 4.4. It is clear that in some cases we can make $\overline{\overline{\bigcup_{a \in M} U^{\prime}(a)}}<\max \left\{\overline{\bar{M}}, \$_{0}\right\}$ in Theorem 4.12 ).

We can get also Löwenheim theorem for p.B. models by the same method as the above.

Theorem 4.6. Let $(P, V)$ be a p.B. model such that $\overline{\bar{P}}<\overline{\bar{V}}$. Then there is a p.B. model $\left(P, V^{\prime}\right)$ such that
1) $L^{+}\left(P, V^{\prime}\right)=L^{+}(P, V)$,
2) $\overline{\bar{V}}^{\prime} \leqq \max \left\{\overline{\bar{P}}, \aleph_{0}\right\}$. 
We have dealt with the cardinality of the universe of a model in the above. On the other hand, can we contract the cardinality of a partially ordered set (or a pseudo-Boolean algebra) of a Kripke (or a p.B.) model without changing the logic characterized by it? In the case of propositional logics, we can show that for any pseudo-Boolean algebra $P$ there exists a pseudo-Boolean algebra $Q$ such that

$$
L^{+}(Q)=L^{+}(P) \text { and } \overline{\bar{Q}} \leqq \$_{0}
$$

by taking the Lindenbaum algebra of $L^{+}(P)$ for $Q .{ }^{1)}$ At present, we can only prove a weak result on p.B. models.

Suppose that $(P, V)$ is a p.B. model such that

$$
\overline{\bar{P}}>\overline{\bar{V}}=\lambda \geqq \kappa_{0}
$$

Write $\lambda^{\prime}=2^{\lambda}$. Let $S$ be any subset of $P$ such that $0_{P} \in S$ and $\overline{\bar{S}} \leqq \lambda$. Corresponding to $S$, define a subset $S_{\mu}$ of $P$ for each ordinal $\mu \leqq \lambda^{\prime}$ by

$$
\begin{aligned}
S_{0}= & S \\
S_{\mu}= & S_{\rho} \cup\left\{\bigcup_{a \in T} a, \bigcap_{a \in T} a ; T \subset S_{\rho} \text { and } T \leqq \lambda\right\} \\
& \cup\left\{a \supset b ; a, b \in S_{\rho}\right\} \quad \text { if } \mu=\rho+1, \\
S_{\mu}= & \bigcup_{\rho<\mu} S_{\rho} \quad \text { if } \mu \text { is a limit ordinal. }
\end{aligned}
$$

Define $P(S)=S_{\lambda^{\prime}}$.

Lemma 4.7. $P(S)$ is a $\lambda$-complete subalgebra of $P$ whose cardinality is not greater than $\lambda^{\prime}$.

Theorem 4.8. For any p.B. model $(P, V)$ such that $\overline{\bar{P}}>\overline{\bar{V}}(=\lambda)$, there is a p.B. model $\left(P^{\prime}, V\right)$ such that

1) $L^{+}\left(P^{\prime}, V\right)=L^{+}(P, V)$,

2) $\overline{\bar{P}}^{\prime} \leqq \kappa$, where $\kappa=\max \left\{\aleph_{0}, \min \left\{\overline{\bar{P}}, \lambda^{\prime}\right\}\right\}$.

1) $L^{+}(P)$ denotes the intermediate propositional logic characterized by $P$. 
Proof. We first remark that if either $\aleph_{0} \leqq \overline{\bar{P}} \leqq \lambda^{\prime}$ or $\overline{\bar{P}} \leqq \$_{0}$ then we have only to take $P$ for $P^{\prime}$. Now, suppose that $\overline{\bar{P}}>\lambda^{\prime}$ and $\lambda \geq \boldsymbol{S}_{0}$. Let $H$ be the set of closed formulas not valid in $(P, V)$. For each formula $A$ in $H$, we choose an assignment $f_{A}$ such that $f_{A}(A) \neq 1_{P}$. Define a subset $S$ of $P$ by

$$
S=\left\{0_{P}\right\} \cup\left\{f_{A}\left(p^{(n)}\left(\bar{v}_{1}, \cdots, \bar{v}_{n}\right)\right) ; A \in H, p^{(n)} \text { is in } \mathscr{L} \text { and } v_{1}, \cdots, v_{n} \in V\right\}
$$

Let $P^{\prime}=P(S)$. Then, $P^{\prime}$ is a $\lambda$-complete subalgebra of $P$ such that $\overline{\bar{P}}^{\prime} \leqq$ $\kappa$ by Lemma 4.7 and each join or meet of at most $\lambda$ elements of $P^{\prime}$ coincides with that of $P$. Thus, we have

$$
L^{+}(P, V) \subset L^{+}\left(P^{\prime}, V\right)
$$

We can show also that $f_{A}(B) \in P^{\prime}$ for any formula $B$ in $\mathscr{L}[V]$ and any $A$ in $H$. In particular, $f_{A}(A) \in P^{\prime}$. Define an assignment $g_{A}$ of $\left(P^{\prime}, V\right)$ by

$$
g_{A}\left(p^{(n)}\left(\bar{v}_{1}, \cdots, \bar{v}_{n}\right)\right)=f_{A}\left(p^{(n)}\left(\bar{v}_{1}, \ldots, \bar{v}_{n}\right)\right) \quad \text { for any } n \text {-ary }(n \geqq 0) \text { predi- }
$$

cate variable $p^{(n)}$ and any $v_{1}, \cdots, v_{n} \in V$.

Then we get $\quad g_{A}(A)=f_{A}(A) \neq 1_{P^{\prime}}$.

Therefore, $\quad L^{+}\left(P^{\prime}, V\right) \subset L^{+}(P, V)$.

Next, let $\lambda<\psi_{0}<\overline{\bar{P}}$. Then we can show that $L^{+}(P, V)=L^{+}\left(P^{*}, V\right)$, where $P^{*}$ is the Lindenbaum algebra of the propositional logic $L^{+}(P)$. Of course, $\overline{\bar{P}}^{*}=\aleph_{0}=\kappa$. This gives our theorem.

\section{§5. Some Syntactical Results}

In the present $\S$, we shall show two syntactical properties of predicate logics, applying the axiomatic method. These results will be used in the next $\S$.

At first, we remark the following fact. If a formula $A$ is in a predicate logic $L+\left\{B_{i} ; i \in I\right\}$ then there is a sequence of formulas $C_{1}, \ldots$, 
$C_{n}(=A)(n \geqq 1)$ such that for each $k \leqq n$,

either 1) $C_{k}=B_{i}$ for some $i \in I$, or $C_{k} \in L$,

or 2) $C_{k}$ is of the form $\forall x C_{i}$ or of the form $\check{S}_{B}^{\left.\not x_{1}, \ldots, x_{m}\right)} C_{i} \mid$ for some $i<k$,

or 3) there are $i, j<k$ such that $C_{j}=\left(C_{i} \rightarrow C_{k}\right)$.

Definition 5.1. An instance of a formula $A$ is defined recursively as follows;

1) $A$ is an instance of $A$,

2) if $B$ is an instance of $A$ then any formula obtained from $B$ by using the substitution or the generalization or the alphabetic change of bound or free variables is an instance of $A$.

It is obvious that if a formula $A$ is in a predicate logic $L$ then any instance of $A$ is in $L$. The following lemma can be easily verified.

Lemma 5.2. If a formula $B$ is in $L+\left\{A_{i} ; i \in I\right\}$ then there are formulas $C_{1}, \ldots, C_{n}$ such that

1) $\left(\bigwedge_{j=1}^{n} C_{j} \rightarrow B\right) \in L$ and

2) each $C_{j}$ is an instance of some $A_{i}$.

Definition 5.3. An instance $B$ of a formula $A$ is said to be elementary, if

either 1) the outermost logical symbol of $B$ is not a universal quantifier,

or 2) $B$ is of the form $\forall x C$ and $C$ is not an instance of $A$.

Let $B$ be an instance of $A$. Then there is a formula $C$ such that $B$ is of the form $\forall x_{1} \cdots \forall x_{n} C$ and $C$ is an elementary instance of $A$. Clearly, such a formula $C$ is determined uniquely by $B$. Each elementary instance of a formula $A$ can be obtained from $A$ without the generalization.

Lemma 5.4 Suppose that $A$ and $B$ are closed formulas having no 
predicate variables in common and that $A_{1}$ and $B_{1}$ are instances of $A$ and $B$, respectively. Then there is an instance $C$ of $A \vee B$ such that

$$
\left(C \rightarrow A_{1} \vee B_{1}\right) \in L J+D i s,
$$

if $A_{1}$ and $B_{1}$ have neither individual nor predicate variables in common.

Proof. Suppose that

$$
\begin{array}{ll}
A_{1}=\forall x_{1} \cdots \forall x_{m} A_{2} & (m \geqq 0) \quad \text { and } \\
B_{1}=\forall y_{1} \cdots \forall y_{n} B_{2} & (n \geqq 0),
\end{array}
$$

where $A_{2}$ and $B_{2}$ are elementary instances of $A$ and $B$, respectively. Put

$$
C=\forall x_{1} \cdots \forall x_{m} \forall y_{1} \cdots \forall y_{n}\left(A_{2} \vee B_{2}\right)
$$

Then we get

$$
\left(C \rightarrow A_{1} \vee B_{1}\right) \in L J+D i s
$$

In addition, we can show that $C$ is an instance of $A \vee B$.

Now we have the following theorem.

Theorem 5.5. Let Dis be in a predicate logic L. Then if closed formulas $A$ and $B$ have no predicate variables in common,

$$
(L+A) \cap(L+B)=L+A \vee B \text {. }
$$

Proof. It is clear that

$$
L+A \vee B C(L+A) \cap(L+B)
$$

Let $F$ be a formula in $(L+A) \cap(L+B)$. Then by Lemma 5.2 , there are instances $C_{i}(1 \leqq i \leqq m)$ of $A$ and instances $D_{j}(1 \leqq j \leqq n)$ of $B$ such that

$$
\left(\bigwedge_{i=1}^{m} C_{i} \rightarrow F\right) \in L \quad \text { and } \quad\left(\bigwedge_{j=1}^{n} D_{j} \rightarrow F\right) \in L
$$

By replacing some predicate and individual variables in 
$\left(\bigwedge_{j=1}^{n} D_{j} \rightarrow F\right.$ ) by other variables not appearing in any of $C_{i}, D_{j}$ and $F$, we can get formulas $D_{j}^{\prime}$ and $F^{\prime}$ such that

1) $\left(\bigwedge_{j=1}^{n} D_{j}^{\prime} \rightarrow F^{\prime}\right) \in L$,

2) for each $i$ and $j, C_{i}$ and $D_{j}^{\prime}$ have neither predicate nor individual variables in common,

3) $F$ can be obtained from $F^{\prime}$ by replacing some predicate and individual variables.

Then, we have that

$$
\left(\bigwedge_{i=1}^{m} \bigwedge_{j=1}^{n}\left(C_{i} \vee D_{j}^{\prime}\right) \rightarrow\left(F \vee F^{\prime}\right)\right) \in L
$$

Since each $D_{j}^{\prime}$ is an instance of $B$, applying Lemma 5.4 we have that for each $i$ and $j$ there is an instance $E_{i j}$ of $A \vee B$ such that

$$
\left(E_{i j} \rightarrow\left(C_{i} \vee D_{j}^{\prime}\right)\right) \in L
$$

Thus,

$$
\left(\left(\bigwedge_{i=1}^{m} \bigwedge_{j=1}^{n} E_{i j}\right) \rightarrow\left(F \vee F^{\prime}\right)\right) \in L
$$

Therefore,

$$
F \vee F^{\prime} \in L+A \vee B
$$

By replacing some predicate and individual variables in $F^{\prime}$, we get

$$
F \vee F \in L+A \vee B
$$

Hence,

$$
F \in L+A \vee B
$$

As we show in the following, it does not hold always that

$$
(L J+A) \cap(L J+B)=L J+A \vee B,
$$


(cf. Theorem $2.1[2]$ ).

Theorem 5.6. $\left(L J+R_{2}\right) \cap(L J+D i s) \neq L J+R_{2} \vee D i s$, where $R_{2}=r \vee$ $(r \rightarrow s) \vee \neg s$.

Proof. Let $R_{2}^{*}=\forall x(r(x) \vee(r(x) \rightarrow s(x)) \vee \neg s(x))$.

Clearly,

$$
R_{2}^{*} \vee D i s \in\left(L J+R_{2}\right) \cap(L J+D i s)
$$

But, we can show that

$$
R_{2}^{*} \vee D i s \notin L J+R_{2} \bigvee D i s,
$$

by using the p.B. model introduced in the proof of Theorem 2.8.

In $§ 6$, we shall study a relation between a predicate logic and its propositional fragment. We prove a theorem which we shall use often in our development in the next .

Suppose that a formula $A$ is given. $\alpha$ is a function from the set of atomic formulas appearing in $A$ to the set of propositional variables such that

1) $\alpha(p)=p$ for any propositional variable $p$,

2) $\alpha\left(p\left(x_{1}, \ldots, x_{n}\right)\right)=\alpha\left(p\left(y_{1}, \ldots, y_{n}\right)\right)$ for any predicate variable $p$ and any individual variables $x_{1}, \cdots, x_{n}, y_{1}, \cdots, y_{n}$,

$3)$ if $\alpha\left(p\left(x_{1}, \cdots, x_{m}\right)\right)=\alpha\left(q\left(y_{1}, \ldots, y_{n}\right)\right) \quad(m, n \geqq 0)$ then $p=q$ (and $m=n$ ).

We extend $\alpha$ by using the following rules;

for any formula $B, C$,

$$
\begin{aligned}
& \alpha(B \wedge C)=\alpha(B) \wedge \alpha(C), \\
& \alpha(B \vee C)=\alpha(B) \vee \alpha(C), \\
& \alpha(B \rightarrow C)=\alpha(B) \rightarrow \alpha(C), \\
& \alpha(\neg B)=\neg \alpha(B), \\
& \alpha(\forall x B)=\alpha(B),
\end{aligned}
$$




$$
\alpha(\exists x B)=\alpha(B) .
$$

It is clear that $\alpha(A)$ is a propositional formula. $\alpha(A)$ is called an associated propositional formula (apf) of $A$. (See Church [1]. The word an associated formula of propositional calculus is used in it.) Taking an enumeration of all the predicate variables, we can assume that the value $\alpha(A)$ is determined uniquely by $A$. Thus, we say henceforth that $\alpha(A)$ is the apf of $A$. We can show that if a formula is in a predicate logic $L$ then its apf is also in $L$ and hence in $\pi(L)$.

Theorem 5.7. For each predicate logic $L$ and each set of formulas $\left\{B_{i} ; i \in I\right\}$,

$$
\pi\left(L+\left\{B_{i} ; i \in I\right\}\right)=\pi(L)+\left\{\alpha\left(B_{i}\right) ; i \in I\right\} .
$$

Proof. As $B_{i} \in L+\left\{B_{i} ; i \in I\right\}$ for $j \in I, \alpha\left(B_{j}\right) \in \pi\left(L+\left\{B_{i} ; i \in I\right\}\right)$. Clearly,

$$
\pi(L) \subset \pi\left(L+\left\{B_{i} ; i \in I\right\}\right) .
$$

Therefore,

$$
\pi(L)+\left\{\alpha\left(B_{i}\right) ; i \in I\right\} \subset \pi\left(L+\left\{B_{i} ; i \in I\right\}\right) .
$$

Suppose that a propositional formula $A$ is in $L+\left\{B_{i} ; i \in I\right\}$. Then by Lemma 5.2, there are formulas $C_{1}, \cdots, C_{n}$ such that $\left(\bigwedge_{j=1}^{n} C_{j} \rightarrow A\right) \in L$ and each $C_{j}$ is an instance of some $B_{n_{j}}$. So, we have that

$$
\alpha\left(\bigwedge_{j=1}^{n} C_{j} \rightarrow A\right)=\bigwedge_{j=1}^{n} \alpha\left(C_{j}\right) \rightarrow A \in \pi(L)
$$

Since each $\alpha\left(C_{j}\right)$ can be obtained from $\alpha\left(B_{n_{j}}\right)$ by using only the substitution of propositional formulas,

$$
A \in \pi(L)+\left\{\alpha\left(B_{i}\right) ; i \in I\right\} .
$$

Corollary 5.8. If a predicate logic $L$ is finitely axiomatizable then $\pi(L)$ is also finitely axiomatizable. 


\section{§6. Propositional Fragments and Predicate Extensions}

Let $K$ be a propositional logic. Then it is interesting to see the structure of the set $\pi^{-1}(K)$. We say that $L$ is a predicate extension of a propositional logic $K$ if $L \in \pi^{-1}(K)$. Of course, $L K$ is the only predicate extension of $L K_{\pi}$. If we look over Umezawa [12] then we know by using Theorem 5.7 that there are many predicate extensions of $L J_{\pi}$. For instance, there is a predicate extension of $L J_{\pi}$ in which every formula is logically equivalent to its prenex normal form.

Theorem 6.1. $\pi$ is a homomorphism preserving all infinite meets and joins in $\mathscr{I}$. That $i s$, for any set of predicate logics $\left\{L_{i} ; i \in I\right\}$,

$$
\begin{aligned}
& \pi\left(\bigcap_{i \in I} L_{i}\right)=\bigcap_{i \in I} \pi\left(L_{i}\right) \quad \text { and } \\
& \pi\left(\bigcup_{i \in I} L_{i}\right)=\bigcup_{i \in I} \pi\left(L_{i}\right) .
\end{aligned}
$$

Theorem 6.2. For any propositional logic $K$, there are the strongest logic and the weakest logic among the predicate extension of $K$.

Proof. Let

$$
\begin{aligned}
& L_{1}=L J+\{A ; A \in L K \text { and } \alpha(A) \in K\} \text { and } \\
& L_{2}=L J+\{A ; A \in K\} .
\end{aligned}
$$

Then it is easy to see that $L_{1}$ is the strongest and $L_{2}$ is the weakest logic of $\pi^{-1}(K)$.

In the following, $K^{*}$ and $K_{*}$ denote the strongest and the weakest logic of $\pi^{-1}(K)$, respectively.

Definition 6.3. Formulas $E x, Y_{m}(x)(m \geqq 1)$ and $Z_{n}(n \geqq 1)$ are defined as follows;

$$
E x=p \vee \neg p,
$$




$$
\begin{aligned}
& Y_{1}(x)=p_{1}(x), \\
& Y_{m}(x)=\left(\bigwedge_{i=1}^{m-1} \neg p_{i}(x)\right) \wedge p_{m}(x) \quad \text { if } m>1, \\
& Z_{n}=\left(\bigwedge_{i=1}^{n} \exists x Y_{i}(x)\right) \rightarrow \forall x\left(\bigvee_{i=1}^{n} Y_{i}(x)\right) .
\end{aligned}
$$

Lemma 6.4. For any propositional logic $K$ and any formula $A$,

$$
\alpha(A) \in K \text { if and only if } A \in K_{*}+Z_{1} \text {. }
$$

The following axiomatization of $K^{*}$ relative to $K_{*}$ gives another characterization of $K^{*}$.

Theorem 6.5. For each propositional logic $K$,

$$
K^{*}=K_{*}+E x \vee Z_{1} \text {. }
$$

Proof. By Lemma 6.4,

$$
\begin{aligned}
K^{*} & =L K \cap\left(K_{*}+Z_{1}\right) \\
& =\left(K_{*}+E x\right) \cap\left(K_{*}+Z_{1}\right) .
\end{aligned}
$$

Since $\operatorname{Dis} \in\left(K_{*}+E x\right) \cap\left(K_{*}+Z_{1}\right)$, we have

$$
K^{*}=K_{*}+D i s+E x \vee Z_{1}
$$

by using Theorem 5.5. However, as $D i s \in L J+E x \vee Z_{1}$,

$$
K^{*}=K_{*}+E x \vee Z_{1} \text {. }
$$

Corollary 6.6 If $K$ is finitely axiomatizable then $K^{*}$ and $K_{*}$ are also finitely axiomatizable.

We notice here that even if $K$ has the disjunction property $(d p) K^{*}$ does not have $d p$. (For the definition of the $d p$, see e.g. [8].) We can show that many predicate extension of a propositional logic having the $d p$ do not have the $d p$. It is interesting to see the relation between the $d p$ and the following property; 
if $\exists x A(x) \in L$ then there is a variable $z$ such that $A(z) \in L$. However, we have very little knowledge of the subject. (Cf. RasiowaSikorski [11].)

Theorem 6.7. For each propositional logic $K, K^{*}$ has a characteristic set of p.B. models.

Proof. We remark that $K^{*}=L K \cap\left(K_{*}+Z_{1}\right)$. It suffices to show that $K_{*}+Z_{1}=L^{+}\left(P_{K},\{0\}\right)$, where $P_{K}$ denotes the Lindenbaum algebra of K. Clearly,

$$
K_{*}+Z_{1} \subset L^{+}\left(P_{K},\{0\}\right)
$$

Let $A \in L^{+}\left(P_{K},\{0\}\right)$. Then, $\alpha(A) \in L^{+}\left(P_{K}\right)=K^{2}{ }^{2)} \quad$ Thus, $A \in K_{*}+Z_{1}$ by Lemma 6.4 .

On the other hand, it remains open whether each $K_{*}$ can be characterized by some models.

Theorem 6.8. Let $K$ and $K^{\prime}$ be propositional logics such that $K C$ $K^{\prime}$. Then there is a surjection from $\pi^{-1}(K)$ to $\pi^{-1}\left(K^{\prime}\right)$. Hence, $\overline{\overline{\pi^{-1}(K)}}$ $\geq \overline{\pi^{-1}\left(K^{\prime}\right)}$.

Proof. Define a function $\psi_{K K^{\prime}}$ from $\pi^{-1}(K)$ to $\pi^{-1}\left(K^{\prime}\right)$ by

$$
\psi_{K K^{\prime}}(L)=L \cup\left(K^{\prime}\right)_{*} \quad \text { for any } L \in \pi^{-1}(K) \text {, }
$$

and a function $\varphi_{K^{\prime} K}$ from $\pi^{-1}\left(K^{\prime}\right)$ to $\pi^{-1}(K)$ by

$$
\varphi_{K^{\prime} K}(L)=L \cap K^{*} \quad \text { for any } L \in \pi^{-1}\left(K^{\prime}\right) \text {. }
$$

We notice that

$$
\begin{aligned}
\phi_{K K^{\prime}}\left(K^{*}\right) & =K^{*} \cup\left(K^{\prime}\right)_{*} \\
& =\left(K_{*}+E x \vee Z_{1}\right) \cup\left(K^{\prime}\right)_{*} \\
& =\left(K^{\prime}\right)_{*}+E x \vee Z_{1}
\end{aligned}
$$

2) Remark that $\pi\left(L^{+}(P, V)\right)=L^{+}(P)$ for any p. B. model $(P, V)$. 


$$
=\left(K^{\prime}\right)^{*},
$$

applying Theorem 6.5. Now, we show in the following that $\psi_{K K^{\prime}}$ is surjective. It suffices to show that $\psi_{K K^{\prime}}\left(\varphi_{K^{\prime} K}(L)\right)=L$ for any predicate $\operatorname{logic} L$ in $\pi^{-1}\left(K^{\prime}\right)$. By the definition,

$$
\begin{aligned}
\phi_{K K^{\prime}}\left(\varphi_{K^{\prime} K}(L)\right) & =\varphi_{K^{\prime} K}(L) \cup\left(K^{\prime}\right)_{*} \\
& =\left(L \cap K^{*}\right) \cup\left(K^{\prime}\right)_{*} \\
& =\left(L \cup\left(K^{\prime}\right)_{*}\right) \cap\left(K^{*} \cup\left(K^{\prime}\right)_{*}\right) \\
& =\left(L \cup\left(K^{\prime}\right)_{*}\right) \cap\left(K^{\prime}\right)^{*} \\
& =L,
\end{aligned}
$$

since $L \in \pi^{-1}\left(K^{\prime}\right)$.

Corollary 6.9. For any predicate extension $L$ of a propositional logic $K$, there is a predicate extension $L^{\prime}$ of $L J_{\pi}$ such that $L=L^{\prime} \cup K_{*}$. Hence, if $L$ is finitely axiomatizable then there are propositional formulas $A_{1}, \cdots$, $A_{m}$ and formulas $B_{1}, \ldots, B_{n}$ such that

$$
\begin{aligned}
& L=L J+A_{1}+\cdots+A_{m}+B_{1}+\cdots+B_{n}, \\
& K=L J_{\pi}+A_{1}+\cdots+A_{m} \text { and } \\
& \alpha\left(B_{i}\right) \in L J_{\pi} \quad \text { for any } i \leqq n .
\end{aligned}
$$

This corollary tells us that the variety of the intermediate predicate logics is nothing but that of the logics in $\pi^{-1}\left(L J_{\pi}\right)$.

Corollary 6.10. The function $\varphi_{K^{\prime} K}$ is an isomorphism from $\pi^{-1}\left(K^{\prime}\right)$ into $\pi^{-1}(K)$, which preserves all infinite meets and joins in $\pi^{-1}\left(K^{\prime}\right)$. That is,

$$
\begin{aligned}
& \varphi_{K^{\prime} K}\left(\bigcap_{i \in I} L_{i}\right)=\bigcap_{i \in I} \varphi_{K^{\prime} K}\left(L_{i}\right) \text { and } \\
& \varphi_{K^{\prime} K}\left(\bigcup_{i \in I} L_{i}\right)=\bigcup_{i \in I} \varphi_{K^{\prime} K^{\prime}}\left(L_{i}\right) \quad \text { hold }
\end{aligned}
$$


for any set of predicate logics $\left\{L_{i} ; i \in I\right\}$ in $\pi^{-1}\left(K^{\prime}\right)$.

Let $S_{2}$ be the 3 -valued linear pseudo-Boolean algebra. We know that the propositional logic $L^{+}\left(S_{2}\right)$ is the strongest logic among the propositional logics weaker than $L K_{\pi}$ (see [2]).

Theorem 6.11. $\pi^{-1}\left(L^{+}\left(S_{2}\right)\right)$ contains at least countable predicate logics. Moreover, there are logics in it which are mutually incomparable.

Proof. It is easy to verify that

$$
\begin{aligned}
L^{+}\left(S_{2}\right)_{*} & \subsetneq L^{+}\left(S_{2}\right)_{*}+E x \vee Z_{n+1} \\
& \varsubsetneqq L^{+}\left(S_{2}\right)_{*}+E x \vee Z_{n} \\
& \varsubsetneqq L^{+}\left(S_{2}\right)^{*} \quad \text { for any } n>1 .
\end{aligned}
$$

Now, let $M$ be a set $\{a, b, c\}$ with the order $\leqq$ such that $a \leqq a, b \leqq b$, $c \leqq c$ and $b \leqq c$ hold and let $U_{1}^{n}$ and $U_{2}^{n}(n>1)$ be functions from $M$ to the power set of the set of non-negative integers such that

$$
\begin{aligned}
& U_{1}^{n}(a)=U_{2}^{n}(a)=\{i ; i<\omega\}, \\
& U_{1}^{n}(b)=\{0\}, \\
& U_{2}^{n}(b)=U_{1}^{n}(c)=U_{2}^{n}(c)=\{i ; i \leqq n\} .
\end{aligned}
$$

Then, $L\left(M, U_{1}^{n}\right)$ and $L\left(M, U_{2}^{n}\right)$ are in $\pi^{-1}\left(L^{+}\left(S_{2}\right)\right)$. But if $n>m$ then $L\left(M, U_{1}^{m}\right)$ is incomparable with $L\left(M, U_{2}^{n}\right)$.

Applying Theorem 6.8, we have the following corollary.

Corollary 6.12. For each propositional logic $K$ weaker than $L K_{\pi}$, there are at least countable predicate extensions of $K$.

The above corollary does not mean that the case of $L K_{\pi}$ is exceptional, since $L K+Z_{n}(n \geqq 1)$ is a (consistent but not intermediate) predicate extension of $L K$.

Theorem 6.13. For each predicate logic $L$ such that 


$$
L \subset L^{+}\left(P_{I},\{0,1\}\right) \cap L K \quad\left(\in \pi^{-1}(L J)\right),
$$

where $P_{I}$ is the Lindenbaum algebra of $L J_{\pi}$, define a function $\theta_{L}$ from the set $\mathscr{I}_{\pi}$ of all the propositional logics into $\pi^{-1}\left(L J_{\pi}\right)$ by

$$
\theta_{L}(K)=\left(K_{*} \cup L\right) \cap\left(L J_{\pi}\right)_{*} \quad \text { for any } K \in \mathscr{I}_{\pi}
$$

Then, 1) $\theta_{L}$ is injective,

2) $\theta_{L}\left(L J_{\pi}\right)=L$ and $\theta_{L}\left(L K_{\pi}\right)=\left(L J_{\pi}\right)^{*}=L J+E x \vee Z_{1} \cdot$

Furthermore, if Dis $\in L$ then $\theta_{L}$ is an isomorphism.

Proof. It can be easily ascertained that

$$
\theta_{L}\left(L J_{\pi}\right)=L \text { and } \theta_{L}\left(L K_{\pi}\right)=\left(L J_{\pi}\right)^{*}
$$

Let $K$ and $K^{\prime}$ be distinct propositional logics. We may suppose $K^{\prime} \not \subset K$. Then we can take a formula $A \in K^{\prime}-K$. Clearly,

$$
A \vee Z_{1} \in \theta_{L}\left(K^{\prime}\right)
$$

Suppose that $\theta_{L}(K)=\theta_{L}\left(K^{\prime}\right)$. Then,

$$
\begin{gathered}
A \vee Z_{1} \in \theta_{L}(K) \subset K_{*} \cup L^{+}\left(P_{I},\{0,1\}\right) \\
\subset L^{+}\left(P_{K},\{0,1\}\right),
\end{gathered}
$$

where $P_{K}$ is the Lindenbaum algebra of $K$. As $Z_{1} \notin L^{+}\left(S_{1},\{0,1\}\right)$, where $S_{1}$ is the 2 -valued Boolean algebra, we can show that

$$
A \in L^{+}\left(P_{K},\{0,1\}\right) \text {. }
$$

But since $A$ is a propositional formula, $A$ must be in $L^{+}\left(P_{K}\right)$. So, $A \in K$. This is a contradiction. Thus $\theta_{L}$ is injective. We remark that $\theta_{L}\left(K \cup K^{\prime}\right)=\theta_{L}(K) \cup \theta_{L}\left(K^{\prime}\right)$ holds always since $\left(K \cup K^{\prime}\right)_{*}=K_{*} \cup\left(K^{\prime}\right)_{*}$. Now we suppose that $D i s \in L$. Then we can prove in the same way as Theorem 5.5 that

$$
\theta_{L}\left(K \cap K^{\prime}\right)=\left(\left(K \cap K^{\prime}\right)_{*} \cup L\right) \cap\left(L J_{\pi}\right)^{*}
$$




$$
\begin{aligned}
& =\left(\left(K_{*} \cup L\right) \cap\left(\left(K^{\prime}\right)_{*} \cup L\right)\right) \cap\left(L J_{\pi}\right)^{*} \\
& =\theta_{L}(K) \cap \theta_{L}\left(K^{\prime}\right) .
\end{aligned}
$$

Corollary 6.14. There are $2 \$ 0$ predicate extensions of $L J_{\pi}$.

Proof. By Theorem 6.13 and Jankov's result [4].

We say that a formula is unary if it contains only propositional and unary predicate variables. $\pi_{1}(L)$ denotes the set of unary formulas in a predicate logic $L$. Now consider the following problem;

can any predicate logic be axiomatized by using only unary formulas? If the answer is affirmative, then for any predicate logic $L$ and $L^{\prime} L \neq L^{\prime}$ implies $\pi_{1}(L) \neq \pi_{1}\left(L^{\prime}\right)$. But this does not hold, as we show in the following.

Lemma 6.15. Let $A$ be any unary formula. Then if $A \notin L^{+}(P,\{i ; i<\omega\}) \quad$ for $\quad a$ finite pseudo-Boolean algebra $P$, $A \notin L^{+}(P,\{i ; i \leqq n\})$ for some $n<\omega$.

Proof. This can be proved similarly as a theorem in Löwenheim [6].

Theorem 6.16. There are predicate logics $L, L^{\prime}$ such that $L \neq L^{\prime}$ but $\pi_{1}(L)=\pi_{1}\left(L^{\prime}\right)$. Hence there is a predicate logic which can not be axiomatized by using (even infinitely many) unary formulas.

Proof. Let

$$
\begin{aligned}
& L=\bigcap_{n<\omega} L^{+}(P,\{i ; i \leqq n\}) \cap L K \text { and } \\
& L^{\prime}=L^{+}(P,\{i ; i<\omega\}),
\end{aligned}
$$

where $P$ is any finite pseudo-Boolean algebra such that $E x \notin L^{+}(P)$. As $E x \vee$ Fin $\in L-L^{\prime}$, we have that $L^{\prime} \varsubsetneqq L$. It follows from this that $\pi_{1}\left(L^{\prime}\right) C$ $\pi_{1}(L)$. On the other hand, $\pi_{1}(L) \subset \pi_{1}\left(L^{\prime}\right)$ by Lemma 6.15. Thus $\pi_{1}(L)$ $=\pi_{1}\left(L^{\prime}\right)$. 


\section{References}

[1] Church, A., Introduction to mathematical logic I, Princeton, N. J., 1956.

[2] Hosoi, T., On intermediate logics I, J. Fac. Sci., Univ. Tokyo, Sec. I, 14 (1967), 293-312.

[3] Hosoi, T. and H. Ono, Intermediate propositional logics (A survey), J. of Tsuda College, 5 (1973).

[4] Jankov, V. A., Constructing a sequence of strongly independent superintuitionistic propositional calculi, Soviet Math. Dokl., 9 (1968), 806-807.

[5] Kripke, S. A., Semantical analysis of intuitionistic logic I, Formal systems and recursive fnnctions, ed. by J. N. Crossley and M. A. Dummett, Amsterdam, 1965, 92-130.

[6] Löwenheim, L., Über Möglichkeiten im Relativkalkül, Math. Ann., 76 (1915), 447470.

[7] Ono, H., Kripke models and intermediate logics, Publ. RIMS, Kyoto Univ., 6 (1970), 461-476.

[8] - Some results on the intermediate logics, Publ. RIMS, Kyoto Univ., 8 (1972), 117-130.

[9] — Incompleteness of semantics for intermediate predicate logics I, Kripke's semantics, to appear.

[10] - Incompleteness of semantics for intermediate predicate logics II, _ The algebraic semantics, to appear.

[11] Rasiowa, H. and R. Sikorski, The mathematics of metamathematics, Monografie Matematyczne, 41, Warszawa, 1963.

[12] Umezawa, T., On logics intermediate between intuitionistic and classical predicate logic, J. Symbolic Logic, 24 (1959), 141-153.

[13] Gabbay, D. M., Applications of trees to intermediate logics, J. Symbolic Logic, 37 (1972), 135-138.

[14] Görnemann, S., A logic stronger than intuitionism, J. Synbolic Logic, 36 (1971), $249-261$. 
